\title{
Development of Functional Markers for Detection of Inactive DFR- $A$ Alleles Responsible for Failure of Anthocyanin Production in Onions (Allium cepa L.)
}

\author{
Jaehyuk Park ${ }^{1}$, Dong Youn $\mathrm{Cho}^{2}$, Jin Seong Moon ${ }^{3}$, Moo-Kyoung Yoon ${ }^{4}$, and Sunggil Kim ${ }^{1 *}$ \\ ${ }^{I}$ Department of Plant Biotechnology, Biotechnology Research Institute, Chonnam National University, Gwangju 500-757, Korea \\ ${ }^{2}$ ONBREETECH Corporation, Haenam 536-803, Korea \\ ${ }^{3}$ Onion Research Institute, Gyeongsangnam-do Agricultural Research \& Extension Services, Changnyeong 635-821, Korea \\ ${ }^{4}$ National Institute of Horticultural \& Herbal Science, Rural Development Administration, Suwon 441-440, Korea
}

\begin{abstract}
Inactivation of the gene coding for dihydroflavonol 4-reductase (DFR) is responsible for the color difference between red and yellow onions (Allium cepa L.). Two inactive $D F R-A$ alleles, $D F R-A^{P S}$ and $D F R-A^{D E L}$, were identified in our previous study. A functional marker was developed on the basis of the premature stop codon that inactivated the $D F R-A^{P S}$ allele. A derived cleaved amplified polymorphic sequences (dCAPS) primer was designed to detect the single nucleotide polymorphism, an $\mathrm{A} / \mathrm{T}$ transition, which produced the premature stop codon. Digested PCR products clearly distinguished the homozygous and heterozygous red $\mathrm{F}_{2}$ individuals. Meanwhile, to develop a molecular marker for detection of the $D F R-A^{D E L}$ allele in which entire $D F R-A$ gene was deleted, genome walking was performed and approximately $3 \mathrm{~kb} \mathrm{5}$ ' and 3 ' flanking sequences of the $D F R-A^{R}$ coding region were obtained. PCR amplification using multiple primers binding to the extended flanking regions showed that more of the extended region of the $D F R-A$ gene was deleted in the $D F R-A^{D E L}$ allele. A dominant simple PCR marker was developed to identify the $D F R-A^{D E L}$ allele using the dissimilar 3' flanking sequences of the DFR-A gene and homologous $D F R-B$ pseudogene. Distribution of the DFR-A $A^{P S}$ and $D F R-A^{D E L}$ alleles in yellow onion cultivars bred in Korea and Japan was surveyed using molecular makers developed in this study. Results showed predominant existence of the $D F R-A^{P S}$ allele in yellow onion cultivars.
\end{abstract}

Additional key words: bulb color, dCAPS marker, genome walking, molecular marker

\section{Introduction}

Bulb color is the major agronomically important trait in onion (Allium cepa L.) breeding programs. Red, yellow, and white bulb colors are common in onion cultivars. More rarely reported colors are gold (Kim et al., 2004b) and chartreuse (El-Shafie and Davis, 1967). Besides the qualitative color differences, a diverse spectrum of quantitatively different bulb colors exists in red and yellow onions. Pigments responsible for onion bulb colors are flavonoid compounds.

Flavonoids are one of the secondary metabolites of plants. Functions include ultraviolet protection, pigmentation, and disease resistance (Dao et al., 2011; Fini et al., 2011; Shirley, 1996). So far, more than 8,000 derivatives of flavonoids have been reported in plants (Veitch and Grayer, 2011) and 54 kinds of flavonoids have been identified in onions (Fossen et al., 1996; Rhodes and Price, 1996; Slimestad et al., 2007). Quercetin is the most abundant flavonoid in onion bulbs, and anthocyanin is the causal pigment of red bulb color. Numerous studies have reported health benefits of flavonoids, such as strong anti-oxidant and anti-cancer activities (Clere et al., 2011; Cook and Samman, 1996; Cushnie and Lamb, 2011; Lotito and Frei, 2006; Nishiumi et al., 2011; Russo et al., 2012).

Biosynthesis pathway of anthocyanin has been extensively studied in model plants such as maize, snapdragon, and petunia (Fig. 1, Holton and Cornish, 1995). Many mutants of color phenotypes of these plant species have been utilized

\footnotetext{
*Corresponding author: dronion@jnu.ac.kr

※ Received 10 July 2012; Revised 4 October 2012; Accepted 10 October 2012. This research was supported by a grant (code: 0636-20110009) from the Vegetable Breeding Research Center through R\&D Convergence Center Support Program and a grant (110046-3) from the Ministry for Food, Agriculture, Forestry, and Fisheries, Korea. Additional support was received from a grant (PJ005407) from the Specific Joint Agricultural Research-Promoting Projects, RDA, Republic of Korea. We thank Ji-wha Hur and Jeong-Ahn Yoo for their dedicated technical help.
} 
to isolate structural genes encoding enzymes involved in the pathway (Ferrer et al., 2008; Vogt, 2010). In addition, multiple regulatory genes controlling expression of whole or part of structural genes in the pathway have been identified (Goodrich et al., 1992; Holton and Cornish, 1995; Quattrocchio et al., 1993; Spelt et al., 2000; Yamazaki et al., 2003). Accumulated sequence information of genes isolated in model plant species have been used in isolating onion genes involved in the anthocyanin biosynthesis pathway and to elucidate the mechanism of onion bulb color inheritance (Kim et al., 2004a, 2005a).

Five major loci involved in inheritance of onion bulb colors have been reported (Clarke et al., 1944; El-Shafie and Davis, 1967; Rieman, 1931). The $I$ and $C$ loci determine white bulb colors. The dominant $I$ allele inhibits color expression resulting in a white bulb color regardless of the genotypes of the other four loci, and the heterozygous genotype of the $I$ locus produces buff or creamy colors (El-Shafie and Davis, 1967). The $C$ locus, which is a basic color factor, is responsible for a white bulb color when the genotype of the $C$ locus is homozygous recessive. The $C$ locus might be a regulatory gene controlling the gene encoding chalcone synthase (CHS), a key enzyme in the flavonoid biosynthesis pathway. Kim et al. (2005b) showed that transcription of two onion $\mathrm{CHS}$ genes was significantly reduced in white $F_{2}$ segregating plants

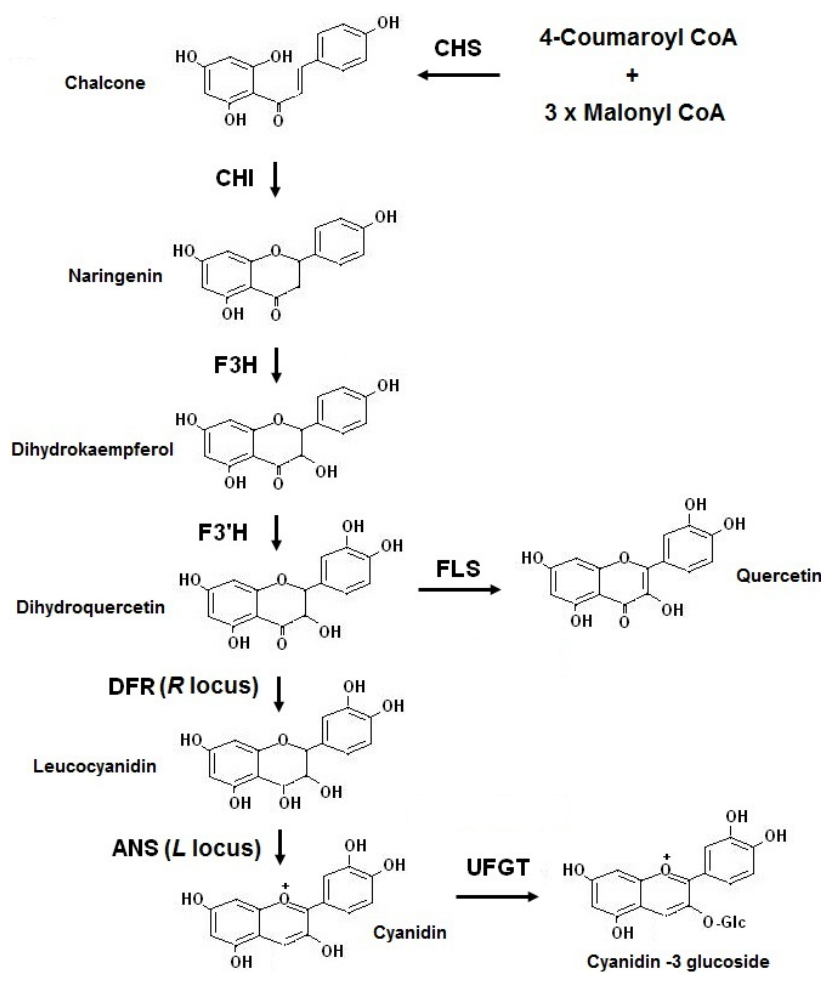

Fig. 1. Anthocyanin biosynthesis pathway in onions. CHS, chalcone synthase; $\mathrm{CHI}$, chalcone isomerase; $\mathrm{F} 3 \mathrm{H}$, flavanone 3-hydroxylase; F3'H, flavonoid 3'-hydroxylase; DFR, dihydroflavonol 4-reductase; ANS, anthocyanidin synthase; FLS, flavonol synthase; UFGT, UDP glucose-flavonoid 3-o-glucosyl transferase. whose genotypes of the $C$ locus were homozygous recessive.

The $G$ locus is responsible for a chartreuse color when the genotype of $I, C$, and $G$ loci is $i i / C$-/gg (El-Shafie and Davis, 1967). Identity of the $G$ locus is still unresolved, but the gene responsible for a gold color, which showed a similar epistatic interaction pattern to that of a chartreuse color, was identified. Inactivation of the gene coding for chalcone isomerase (CHI) due to presence of a premature codon leads to the production of a gold color (Kim et al., 2004b). Since CHI enzyme acts in the next step of CHS in the anthocyanin biosynthesis pathway and catalyzes the conversion of chalcone into naringenin, accumulated chalcone might be the pigment for the gold color.

The $R$ and $L$ loci are complementarily involved in production of a red bulb color. At least one dominant allele of both loci is required to produce a red bulb color (El-Shafie and Davis, 1967). The $R$ and $L$ loci are genes encoding dihydroflavonol 4-reductase (DFR) and anthocyanidin synthase (ANS), respectively (Kim et al., 2005a, 2005c). Inactivation of the DFR gene caused by deletion of 3' coding sequences determines the color difference between yellow and red onions (Kim et al., 2005c). Meanwhile, inactivation of ANS gene due to the presence of a critical amino acid change in the conserved region have been identified in yellow onions grown in Brazil (Kim et al., 2005a). A $F_{1}$ hybrid between the yellow onions containing the inactive $D F R$ gene and the Brazilian yellow onions containing the inactive ANS gene produced a red bulb color by complementation, and the $F_{2}$ population showed a typical 9:7 ratio of red to yellow onions (Kim et al., 2005a).

Two homologous DFR genes were also isolated, but they were known to be pseudogenes. To distinguish the active $D F R$ gene from other homologous genes, active $D F R$ gene was designated $D F R-A$, and two other homologs were named $D F R-B$ and $D F R-C$ (Kim et al., 2005c). Since $D F R-A, D F R-B$, and $D F R-C$ genes were all detected in a doubled haploid line (Kim et al., 2005c), both $D F R-B$ and $D F R-C$ were separate loci, not inactive alleles of the $D F R-A$ gene. The inactive $D F R-A$ allele isolated from yellow onions were designated $D F R-A^{T R N}$. Two additional inactive $D F R-A$ alleles were identified in onion cultivars bred in Korea and Japan, and they were designated $D F R-A^{P S}$ and $D F R-A^{D E L}$ alleles (Kim et al., 2009). The $D F R-A^{P S}$ allele contained a premature stop codon and entire $D F R-A$ gene was deleted in the $D F R-A^{D E L}$ allele.

In this study, we developed an ideal functional marker for detection of the $D F R-A^{P S}$ allele based on the sequence motif creating the premature stop codon and a simple PCR marker for faithful detection of the $D F R-A^{D E L}$ allele. In addition, distribution of inactive $D F R-A$ alleles was surveyed using developed molecular markers in onion cultivars grown in Korea and Japan. 


\section{Materials and Methods}

\section{Plant Materials}

Two $F_{2}$ populations originating from the crosses between red and yellow breeding lines were selected from $11 \mathrm{~F}_{2}$ populations produced in a previous study (Kim et al., 2009). After polymerase chain reaction (PCR) amplification and sequencing of the $D F R-A$ genes of yellow $\mathrm{F}_{2}$ plants, the $\mathrm{F}_{2}$ populations containing the $D F R-A^{P S}$ and $D F R-A^{D E L}$ alleles were selected. A total of 38 onion cultivars recently bred in Korea and Japan were used to survey the frequency of the inactive $D F R-A$ alleles. Cultivar names and the $D F R-A$ alleles are provided in Table 1.

Table 1. Yellow onion cultivars used in this study.

\begin{tabular}{|c|c|c|}
\hline Names & DFR-A alleles & Comments \\
\hline Sura ball & $D F R-A^{P S}$ & \\
\hline Gumgu & $D F R-A^{P S}$ & \\
\hline Tobagi & $D F R-A^{P S}$ & \\
\hline Singihanyangpa & $D F R-A^{P S}$ & \\
\hline Whangjae & $D F R-A^{P S}$ & \\
\hline Chunjudaego & $D F R-A^{P S}$ & \\
\hline Superio & $D F R-A^{P S}$ & \\
\hline Doongji & $D F R-A^{P S}$ & \\
\hline Yamujin & $D F R-A^{P S}$ & \\
\hline New marus & $D F R-A^{P S}$ & \\
\hline Bisulwhang & $D F R-A^{P S}$ & \\
\hline Arus & $D F R-A^{P S}$ & \\
\hline e-Joeun & $D F R-A^{P S}$ & \\
\hline Hanbit515 & $D F R-A^{P S}$ & \\
\hline Sisadul530 & $D F R-A^{P S}$ & \\
\hline Hard ball & $D F R-A^{P S}$ & \\
\hline Sun power & $D F R-A^{P S}$ & \\
\hline Hanrodakari & $D F R-A^{P S}$ & \\
\hline Top ball & $D F R-A^{P S}$ & \\
\hline Dark horse & $D F R-A^{P S}$ & \\
\hline Magic gold & $D F R-A^{P S}$ & \\
\hline Okay & $D F R-A^{P S}$ & \\
\hline Phantom & $D F R-A^{P S}$ & \\
\hline Okimaru & $D F R-A^{P S}$ & \\
\hline Wangjungwang & $D F R-A^{P S}$ & \\
\hline $\mathrm{Su}$ & $D F R-A^{P S}$ & \\
\hline Kreo & $D F R-A^{P S}$ & \\
\hline Chairman & $D F R-A^{P S}$ & \\
\hline Matrix & $D F R-A^{P S}$ & \\
\hline Big ho & $D F R-A^{P S}$ & \\
\hline Daechoowhang & $D F R-A^{P S}$ & \\
\hline Hiromaru & $D F R-A^{P S}$ & \\
\hline Long power & & Uncharacterized allele $+D F R-A^{P S}$ \\
\hline Topase & & Uncharacterized allele $+D F R-A^{P S}$ \\
\hline This & & Uncharacterized allele \\
\hline Marusino & $D F R-A^{D E L}$ & \\
\hline Pachongwhang & $D F R-A^{D E L}$ & \\
\hline Goldangango & $D F R-A^{D E L}$ & \\
\hline
\end{tabular}


DNA Extraction, PCR Amplification, and Sequencing of PCR Products

Total genomic DNA was extracted from the leaves of 4-5 leaf stage seedlings or sprouted bulbs using a cetyl trimethylammonium bromide (CTAB)-based method (Doyle and Doyle, 1987). PCR was performed in a $10 \mu \mathrm{L}$ reaction mixtures containing $0.05 \mu \mathrm{g}$ template, $1 \mu \mathrm{L} 10 \times$ PCR buffer, $0.2 \mu \mathrm{L}$ forward primer $(10 \mu \mathrm{M}), 0.2 \mu \mathrm{L}$ reverse primer $(10 \mu \mathrm{M}), 0.2 \mu \mathrm{L}$ dNTPs (10 mM each), and $0.1 \mu \mathrm{L}$ Advantage 2 polymerase mix (Clontech, Palo Alto, CA). PCR amplification for genotyping the derived cleaved amplified polymorphic sequence (dCAPS) marker was performed with an initial denaturation at $95^{\circ} \mathrm{C}$ for $4 \mathrm{~min}, 10$ cycles of $95^{\circ} \mathrm{C}$ for $30 \mathrm{~s}, 67^{\circ} \mathrm{C}\left(0.8^{\circ} \mathrm{C}\right.$ decrements in each cycle) for $30 \mathrm{~s}$, and $72^{\circ} \mathrm{C}$ for $1 \mathrm{~min}, 35$ cycles of $95^{\circ} \mathrm{C}$ for $30 \mathrm{~s}, 59^{\circ} \mathrm{C}$ for $30 \mathrm{~s}$, and $72^{\circ} \mathrm{C}$ for $1 \mathrm{~min}$, and a final 7-min extension at $72^{\circ} \mathrm{C}$. The PCR products were digested with $\mathrm{Xba \textrm {I }}$ restriction enzyme for $3 \mathrm{~h}$ at $37^{\circ} \mathrm{C}$. The digested PCR products were visualized on $9 \%$ polyacrylamide gels after ethidium bromide staining.

PCR amplification for detection of the $D F R-A^{D E L}$ allele was performed with an initial denaturation at $95^{\circ} \mathrm{C}$ for 5 min, 40 cycles of $95^{\circ} \mathrm{C}$ for $30 \mathrm{~s}, 68^{\circ} \mathrm{C}$ for $30 \mathrm{~s}$, and $72^{\circ} \mathrm{C}$ for $2 \mathrm{~min}$, and a final $10-\mathrm{min}$ extension at $72^{\circ} \mathrm{C}$. The PCR products were visualized on $1.5 \%$ agarose gels after ethidium bromide staining. The primer sequences of the molecular markers developed in this study are presented in Table 2. For sequencing of PCR products, the PCR products were purified using a QIAquick PCR purification kit (QIAGEN, Valencia, CA) and directly sequenced. Sequencing reactions were carried out using Big Dye (Applied Biosystems, Foster City, CA) according to the manufacturer's protocol and the sequences were obtained using an ABI PRISM 3730XL Analyzer (Applied Biosystems).

Identification of the Flanking Sequences of the DFR-A Gene Using Genome Walking

The flanking sequences of the $D F R-A$ gene were obtained using a Universal GenomeWalker Kit (Clontech) according to the manufacturer's protocol. A red breeding line containing the $D F R-A^{R}$ allele was used for construction of genome walking libraries. The amplified PCR products were purified using a QIAquick PCR Purification kit (QIAGEN) followed by sequencing as previously mentioned.

\section{Primer Design for Development of a dCAPS Marker}

A reverse primer of the molecular marker for detection of the $D F R-A^{P S}$ allele was designed using a web-based software, dCAPS Finder 2.0 (Neff et al., 2002). The option for one mismatch introduction in the primer was selected. As suggested by the manual of dCAPS Finder software, a forward primer was designed approximately 200 bp upstream of the reverse dCAPS primer using Primer3 v.4.0 software (Rozen and Skaletsky, 2000).

\section{Results}

Development of a Functional Marker for Selection of the $D F R-A^{P S}$ Allele

An inactive $D F R-A$ allele, $D F R-A^{P S}$, harboring a premature stop codon was identified from yellow onion cultivars bred in Korea and Japan in a previous study (Kim et al., 2009). A 20-bp deletion in the 5 ' non-coding region was initially used for detection of the $D F R-A^{P S}$ allele, but this 20-bp deletion in the $D F R-A^{P S}$ allele was also found in another active DFR- $A$ allele (GenBank accession: AY221250) identified from several red onion breeding lines or cultivars (data not shown). Since the 20-bp deletion was identified in an active $D F R-A$ allele, application of the molecular marker based on this deletion should be limited in specific populations and detection of the $D F R-A^{P S}$ allele from unknown onion germplasm would be difficult with this marker.

To improve the limited application of the previously developed molecular marker, attempts were made to develop a functional marker on the basis of the premature stop codon, which was the causal mutation responsible for inactivation of the $D F R-A^{P S}$ allele (Fig. 2). To detect the single nucleotide polymorphism (SNP) producing the premature stop codon, 'TAA', a dCAPS marker was developed. A reverse primer containing one mismatch was designed using the dCAPS Finder software. Introduction of a mismatched ' $G$ ' sequence produced a recognition sequence for the $X b a \mathrm{I}$ restriction enzyme only in the $D F R-A^{P S}$ allele (Fig. 3A). When the forward primer was designed without considering the sequences of two homologous pseudogenes (DFR-B and DFR-C), multiple PCR products were amplified,

Table 2. Primer sequences of molecular markers used in this study. The positions of primers are depicted in Figs. 1 and 3 .

\begin{tabular}{lcc}
\hline \hline Primer names & Primer sequences (5' to 3') & Tm \\
\hline DFR-PS-F & GAATGTTAAGCATAATGAAGTCTTGTAAG & 63.4 \\
DFR-PS-R & AATATGTTTACCCATCCTGTCATCT & 60.9 \\
DFR-DEL-F1 & TGCAAACAGCAGTGGAGCTGCAACTTA & 65.0 \\
DFR-DEL-R1 & TGGCCAACAGTATTGCTTCTTCCCAGT & 65.0 \\
DFR-DEL-R2 & ACGCTGGCTTTCTCATGCTTCTTGAGC & 66.5
\end{tabular}



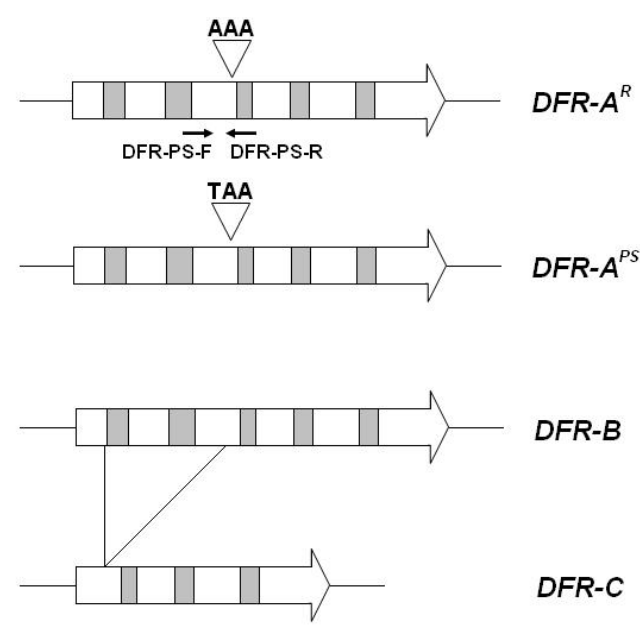

$1 \mathrm{~kb}$

Fig. 2. Organization of the $D F R-A^{R}, D F R-A^{P S}$ alleles, and two homologous pseudogenes. The gray and empty boxes in the coding regions indicate introns and exons, respectively. Arrowshaped boxes indicate the 5'-to-3' direction. The nucleotide sequences on the inverted triangles in the coding region of the DFR-A alleles indicate the single nucleotide polymorphism in the premature stop codon. Arrows indicate primer binding sites.

A

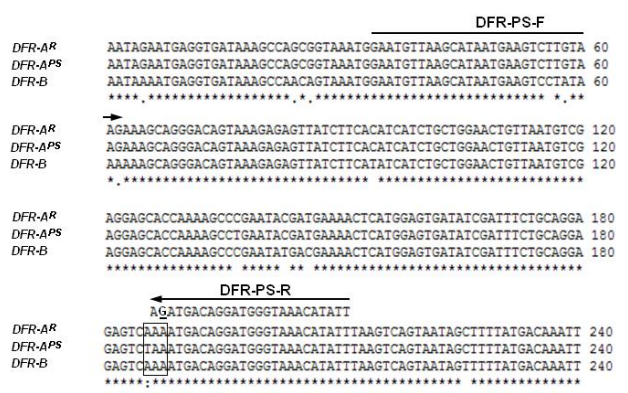

B

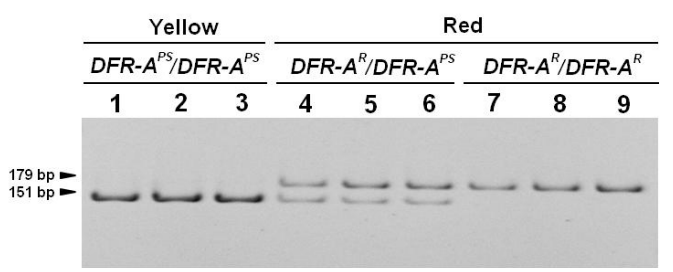

Fig. 3. Development of a functional marker for detection of the $D F R-A^{P S}$ allele. A. Alignment of nucleotide sequences of the $D F R-A^{P S}, D F R-A^{R}$, and DFR-B genes. Only the partial sequences around the premature stop codon are shown. Arrows indicate positions of the forward and reverse primers. Reverse complement sequences of the reverse dCAPS primer are shown under the arrow. The introduced mismatched nucleotide is underlined. The aligned nucleotides including the premature stop codon are enclosed with an empty box. '*' indicates identical nucleotides and both ':' and '?' indicate conservation between groups of strongly and weakly similar properties, respectively. B. Digested PCR products amplified using the primer combination of DFRPS-F and DFR-PS-R. 1-3: yellow $F_{2}$ plants, 4-6: red heterozygous $F_{2}$ plants, 7-9: red homozygous $F_{2}$ plants. although only one band appeared on the agarose gel (data not shown). Both $D F R-A$ and $D F R-B$ genes contains the same number of exons and introns and nucleotide sequence identity between two genes was reported to be $95 \%$ (Kim et al., 2005c). Amplification of multiple PCR products prevented detection of heterozygous individuals from homozygous red $\mathrm{F}_{2}$ plants. Therefore, to avoid amplification of $D F R-B$ and $D F R-C$ genes, a forward primer was designed in the 499-bp deleted region of the $D F R-C$ gene and in the region containing three mismatches between $D F R-A$ and $D F R-B$ genes at the 3' end of primer sequences (Figs. 1 and 2A). A single PCR product was amplified using the improved primer combination, and homozygous and heterozygous red $\mathrm{F}_{2}$ plants were clearly distinguished after digestion of PCR products with the $X b a \mathrm{I}$ restriction enzyme (Fig. 3B). Hereafter, the molecular marker for selection of the $D F R-A^{P S}$ allele is designated as the 'DFR-PS' marker.

Development of a Simple PCR Marker for Detection of the $D F R-A^{D E L}$ Allele

The third inactive $D F R-A$ allele in which the entire $D F R-A$ coding region might be deleted was previously reported (Kim et al., 2009). To obtain more extended flanking sequences of the DFR-A coding regions, multiple cycles of genome walking were carried out, and an 839-bp additional 5' end sequence of the previously reported DFR-A sequence (AY221250) and 2,642-bp 3' end sequences were newly obtained. In addition, the 1,205-bp 3' end sequence of the DFR-B gene was inadvertently acquired during genome walking of the $D F R-A$ gene. The 3' end sequences of the DFR-A and DFR-B genes were completely different starting from the 269-bp downstream of the stop codon except for 211-bp homologous blocks (Fig. 4A). The sequence identity of these blocks between the DFR-A and DFR-B was $89 \%$.

PCR amplification was carried out using multiple primer combinations designed across the extended $D F R$ - $A$ sequences (Fig. 4A). No positive PCR product was observed in yellow $\mathrm{F}_{2}$ bulks containing the DFR-A $A^{D E L}$ allele (Fig. 4B). This result implied that more extended regions might be deleted in the $D F R-A^{D E L}$ allele. Although we failed to obtain the $D F R-A^{D E L}$ specific sequence via genome walking, the 3' extended sequences of the $D F R-A$ and $D F R-B$ genes were used to develop a molecular marker for detection of the $D F R-A^{D E L}$ allele. A common forward primer binding to the homologous sequences of DFR-A and DFR-B genes was designed. The forward primer contained one mismatches at the 3' end of the primer sequences with the $D F R-B$ sequences, but the primer sequences were perfectly matched with the $D F R-A$ sequences. Two reverse primers binding to unique 3' regions of the $D F R-A$ and $D F R-B$ were designed. The presence of one mismatched sequence 
A

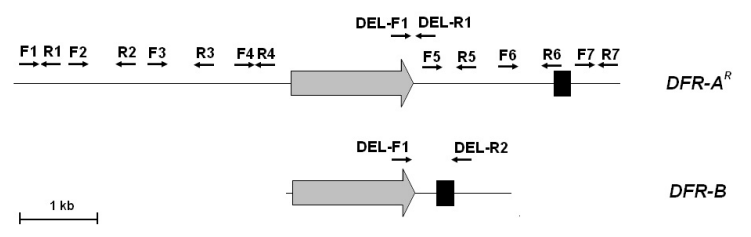

B

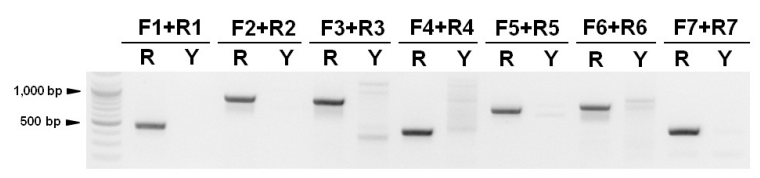

C

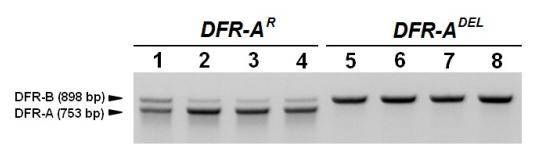

Fig. 4. Development of a simple PCR marker for detection of the $D F R-A^{D E L}$ allele. A. Organization of the flanking sequences of the $D F R-A^{R}$ and $D F R-B$ genes. Arrow-shaped boxes indicate the 5'-to-3' direction and the coding regions including introns. The filled boxes on the 3' end of the DFR genes indicate homologous sequence blocks. B. PCR products amplified using primer combinations binding across the DFR-A $A^{R}$ allele. F1-F7: forward primers, R1-R7: reverse primers. The positions of primers are shown in Fig. 4A. R: red $F_{2}$ bulk, $Y$ : yellow $F_{2}$ bulk. C. PCR products amplified with a combination of three primers (DFR-DEL-F, DFR-DEL-R1, DFR-DEL-R2). 1-4: red $F_{2}$ plants, 5-8: yellow $F_{2}$ plants.

in the forward primer ensured that amplification of the $D F R-B$ sequence was possible if only the $D F R-A^{D E L}$ allele existed, as in the case of yellow onions consisting of homozygous $D F R-A^{D E L}$ alleles. Results of PCR amplification showed that only the $D F R-B$ specific sequence could be amplified in the yellow $\mathrm{F}_{2}$ individuals containing the $D F R-A^{D E L}$ alleles, but a smaller intense band amplified from the $D F R-A$ sequence and a larger faint band amplified from the $D F R-B$ sequences were observed in Red $F_{2}$ individuals (Fig. 4C). This molecular marker was designated the 'DFR-DEL' marker.

\section{Distribution of the DFR-A Alleles in Yellow Onion Cultivars Bred in Korea and Japan}

To test applicability of the newly developed markers and to estimate frequency of the $D F R-A^{P S}$ and $D F R-A^{D E L}$ alleles among yellow onion cultivars bred in Korea and Japan, the $D F R-A$ genotypes of 38 onion cultivars were identified using the DFR-PS and DFR-DEL markers. First, cultivars were screened with the DFR-PS marker to detect the $D F R-A^{P S}$ allele. A majority of the cultivars (84\%) contained the $D F R-A^{P S}$ allele (Table 1). Next, cultivars that did not contain the DFR-A $A^{P S}$ allele were tested with the molecular marker for detection of the $D F R-A^{T R N}$ allele (Kim et al., 2005c). No PCR product corresponding to the $D F R-A^{T R N}$ allele was observed in all tested cultivars (data not shown). This result implied that no cultivar contained the $D F R-A^{T R N}$ allele and that they might contain the DFR-A $A^{D E L}$ allele. The presence of the DFR-A $A^{D E L}$ allele was confirmed by the DFR-DEL marker in three cultivars. As expected, the $D F R-B$ specific PCR products were observed in these three cultivars (data not shown). However, three other cultivars containing an uncharacterized $D F R-A$ allele were also identified. There were two SNPs and one 3-bp insertion on the coding sequences of the uncharacterized allele compared with the $D F R-A^{R}$ allele. Further characterization of this new allele is required in the future study.

\section{Discussion}

Development of a Functional Marker for Detection of the $D F R-A^{P S}$ Allele

A dCAPS marker was developed in this study to detect the $D F R-A^{P S}$ allele containing a premature stop codon. Since this molecular marker was designed on the basis of the single point mutation, in which the 'TAA' stop codon was created by a $\mathrm{A} / \mathrm{T}$ transition, this marker is considered to be a typical 'functional marker' (Andersen and Lübberstedt, 2003). By the definition proposed by Andersen and Lübberstedt (2003), the functional marker is a DNA marker derived from functionally characterized sequence motifs. Because of complete linkage between polymorphic sequence motifs and phenotypic variations, a functional marker is the most ideal marker to be utilized in marker-assisted selection.

On the contrary, the previously developed molecular marker for detection of the $D F R-A^{P S}$ allele developed on the basis of the 20-bp indel located at the 5' nod-coding sequence (Kim et al., 2009) is considered to be a gene-targeted marker according to the definition by Andersen and Lübberstedt (2003). Despite the tight linkage between markers and mutations affecting phenotypic variation, a gene-targeted marker has limitations compared with a functional marker. The number of alleles of a gene-targeted marker is not always matched with the number of alleles of the target gene. Indeed, the 20-bp deletion on the 5' non-coding region of the $D F R-A$ gene was also identified in the other $D F R-A$ alleles other than the $D F R-A^{P S}$ allele. For this reason, only the functional marker could be used in the selection of yellow onion cultivars containing the $D F R-A^{P S}$ allele among unknown onion germplasm. Therefore, the functional marker developed in this study would be more useful in onion breeding programs than the gene-targeted marker.

In particular, a functional marker is useful in marker- 
assisted backcrossing programs as a foreground selection marker (Neeraja et al., 2007; Septiningsih et al., 2009). If a molecular marker linked to the target trait is used as a foreground selection marker, phenotypic confirmation of the selected individuals may be required at least once throughout backcross generations due to the recombination between marker and target gene. In contrast, phenotypic confirmation is not required when a functional marker is used as a foreground selection marker. Therefore, the functional marker for selection of the $D F R-A^{P S}$ allele would be useful in marker-assisted backcrossing programs in onions. For example, desirable red onion breeding lines or cultivars can be bred by transferring the active $D F R-A^{R}$ allele into yellow elite lines or cultivars using marker-assisted backcrossing in which the DFR-PS marker is used as a foreground selection marker. In fact, a maker-assisted backcrossing program for the purpose of improving low storability of red onion cultivars is currently underway in our onion breeding programs. Red onion cultivars bred in Korea were generally poor in storability compared with yellow onion cultivars (Nam et al., 2011).

\section{Utilization of Molecular Markers for Selection of the $D F R-A$ Allele in Onion Breeding Programs}

Utility of functional markers partially depends on robust marker assay technologies. The dCAPS analysis was used in this study to detect the SNP in the $D F R-A^{P S}$ allele. Although SNPs are the most abundant polymorphism in eukaryotic genomes, the discovery of SNPs has been the bottleneck in non-model plant species for which genomics information is limited. However, as sequencing technologies have advanced in recent years (Edward and Batley, 2010; Varshney et al., 2009), numerous SNPs have been generated in many crops. In parallel, many SNP genotyping technologies such as singlebase extension, allele-specific PCR, and pyrosequencing have been developed (Appleby et al., 2009; Chen and Sullivan, 2003). However, most of these technologies have not been widely used in breeding programs due to requirement of expensive equipment, special reagents, or sophisticated protocols. Meanwhile, the dCAPS analysis introduced by Neff et al. (1998) is a relatively simple, inexpensive, and moderate throughput SNP genotyping technique. The web-based software dCAPS Finder 2.0 (Neff et al., 2002) helps to design dCAPS primers for detecting any SNP. Indeed, there was no recognition site for any restriction enzymes in the SNP, which created the premature stop codon in the $D F R-A^{P S}$ allele, but the reverse primers designed by software contained one mismatch and generated a recognition site for the $X b a \mathrm{I}$ restriction enzyme. Taken together, the data indicate that dCAPS analysis is a robust SNP genotyping technique, especially for detecting SNPs affecting phenotypic variations.
The DFR-DEL marker was developed to detect yellow onion cultivars containing the $D F R-A^{D E L}$ allele. However, this marker is limited in detecting heterozygous plants. Since the selection of homozygous red onions from segregating populations is the most important use of molecular markers developed for $D F R-A$ allelic selection, the DFR-DEL marker seems to be useless for this purpose. However, the DFR-DEL marker is useful to detect the presence of the $D F R-A^{D E L}$ allele among unknown onion germplasm. Although the color phenotypes of unknown germplasm are same as yellow, there would be three completely different inactive $D F R-A$ alleles in diverse germplasm. Therefore, identification of specific $D F R-A$ alleles would be a crucial step in bulb color selection of the onion breeding programs. To improve the utility of the DFR-DEL marker, flanking sequences of the deleted $D F R-A$ gene must be obtained. We failed to obtain the flanking sequences using repeated cycles of short-distance genome walking. Therefore, large-scale sequencing of onion genome may be required to acquire flanking sequences of the $D F R-A$ gene in the $D F R-A^{D E L}$ allele.

\section{Literature Cited}

Andersen, J.R. and T. Lübberstedt. 2003. Functional markers in plants. Trends Plant Sci. 11:554-560.

Appleby, N., D. Edwards, and J. Batley. 2009. New technologies for ultra-high throughput genotyping in plants. Methods Mol. Biol. 513:19-39.

Chen, X. and P.F. Sullivan. 2003. Single nucleotide polymorphism genotyping: Biochemistry, protocol, cost and throughput. Pharmacogenomics J. 3:77-96.

Clarke, A.E. and H.A. Jones, and T.M. Little. 1944. Inheritance of bulb color in the onion. Genetics 29:569-575.

Clere, N., S. Faure, M.C. Martinez, and R. Andriantsitohaina. 2011. Anticancer properties of flavonoids: Roles in various stages of carcinogenesis. Cardiovasc. Hematol. Agents Med. Chem. 9:62-77.

Cook, N.C. and S. Samman. 1996. Flavonoids-chemistry, metabolism, cardioprotective effects, and dietary sources. Nutr. Biochem. 7:66-76.

Cushnie, T.P. and A.J. Lamb. 2011. Recent advances in understanding the antibacterial properties of flavonoids. Int. J. Antimicrob. Agents 38:99-107.

Dao, T.T.H., H.H.M. Linthorst, and R. Verpoorte. 2011. Chalcone synthase and tis functions in plant resistance. Phytochem. Rev. 10:397-412.

Doyle, J.J. and J.L. Doyle. 1987. A rapid DNA isolation procedure for small quantities of fresh leaf tissue. Phytochem. Bull. 19:11-15.

Edwards, D. and J. Batley. 2010. Plant genome sequencing: applications for crop improvement. Plant Biotechnol. J. 8:2-9.

El-Shafie, M.W. and G.N. Davis. 1967. Inheritance of bulb color in the onion (Allium cepa L.). Hilgardia 38:607-622.

Ferrer, J.L., M.B. Austin, C. Stewart, Jr., and J.P. Noel. 2008. 
Structure and function of enzymes involved in the biosynthesis of phenylpropanoids. Plant Physiol. Biochem. 46:356-370.

Fini, A., C. Brunetti, M. Di Ferdinando, F. Ferrini, and M. Tattini. 2011. Stress-induced flavonoid biosynthesis and the antioxidant machinery of plants. Plant Signal Behav. 6:709-711.

Fossen, T., O.M. Andersen, D.O. Ovstedal, A.T. Pedersen, and A. Raknes. 1996. Characteristic anthocyanin pattern from onions and other Allium spp. J. Food Sci. 61:703-706.

Goodrich, J., R. Carpenter, and E.S. Coen. 1992. A common gene regulates pigmentation pattern in diverse plant species. Cell 68:955-964.

Holton, T.A. and E.C. Cornish 1995. Genetics and biochemistry of anthocyanin biosynthesis. Plant Cell 7:1070-1083.

Kim, S., D. Baek, D.Y. Cho, and M. Yoon. 2009. Identification of two novel inactive $D F R-A$ alleles responsible for failure to produce anthocyanin and development of a simple PCRbased molecular marker for bulb color selection in onion (Allium cepa L.) Theor. Appl. Genet. 118:1391-1399.

Kim, S., M. Binzel, K. Yoo, S. Park, and L.M. Pike. 2004a. Inactivation of DFR (dihydroflavonol 4-reductase) gene transcription results in blockage of anthocyanin production in yellow onions (Allium cepa). Mol. Breed. 14:253-263.

Kim, S., R. Jones, K. Yoo, and L.M. Pike. 2004b. Gold color in onions (Allium cepa): A natural mutation of the chalcone isomerase gene resulting in a pre-mature termination codon. Mol. Gen. Genomics 272:411-419.

Kim, S., R. Jones, K. Yoo, and L.M. Pike. 2005a.. The $L$ locus, one of complementary genes required for anthocyanin production in onions (Allium cepa), encodes anthocyanidin synthase. Theor. Appl. Genet. 111:120-127.

Kim, S., K. Yoo, and L.M. Pike. 2005b. The basic color factor, the $C$ locus, encodes a regulatory gene controlling transcription of chalcone synthase genes in onions (Allium cepa). Euphytica 142:273-282.

Kim, S., K. Yoo, and L.M. Pike. 2005c. Development of a PCR-based marker utilizing a deletion mutation in the DFR (dihydroflavonol 4-reductase) gene responsible for the lack of anthocyanin production in yellow onions (Allium cepa). Theor. Appl. Genet. 110:588-595.

Lotito, S.B. and B. Frei. 2006. Consumption of flavonoids-rich foods and increased plasma antioxidant capacity in humans: cause, consequence, or epiphenomenon? Free Radic. Biol. Med. 41:1727-1746.

Nam, E., D.Y. Cho, E. Lee, C. Kim, H. Han, M. Yoon, and S. Kim. 2011. Bulb storability of red and yellow onion (Allium cepa L.) cultivars grown in Korea. Kor. J. Breed. Sci. 43: $132-138$.

Neeraja, C.N., R. Maghirang-Rodriguez, A. Pamplona, S. Heuer, B.C.Y. Collard, E.M. Septiningsih, G. Vergara, D. Sanchez, K. Xu, A.M. Ismail, and D.J. Mackill. 2007. A marker-assisted backcross approach for developing submergence-tolerant rice cultivars. Theor. Appl. Genet. 115:767-776.

Neff, M.M., J.D. Neff, J. Chory, and A.E. Pepper. 1998. dCAPS, a simple technique for the genetic analysis of single nucleotide polymorphisms: Experimental applications in Arabidopsis thaliana genetics. Plant J. 14:387-392.

Neff, M.M., E. Turk, and M. Kalishman. 2002. Web-based primer design for single nucleotide polymorphism analysis. Trends Genet. 18:613-615.

Nishiumi, S., S. Miyamoto, K. Kawabata, K. Ohnishi, R. Mukai, A. Murakami, H. Ashida, and J. Terao. 2011. Dietary flavonoids as cancer-preventive and therapeutic biofactors. Front. Biosci. 3:1332-1362.

Quattrocchio, F., J.F. Wing, H.T.C. Leppen, J.N. Mol, and R.E. Koes. 1993. Regulatory genes controlling anthocyanin pigmentation are functionally conserved among plant species and have distinct sets of target genes. Plant Cell 5:1497-1512.

Reiman, G.H. 1931. Genetic factors for pigmentation in the onion and their relation to disease resistance. J. Agr. Res. 42:251-278.

Rhodes, M.J.C. and K.R. Price. 1996. Analytical problems in the study of flavonoid compounds in onions. Food Chem. 57:113-117.

Rozen, S. and H.J. Skaletsky. 2000. Primer3 on the WWW for general users and for biologist programmers, p. 365-386. In: S. Krawetz and S. Misener (eds.). Bioinormatics methods and protocols: Methods in molecular biology. Humana Press, Totowa, NJ.

Russo, M., C. Spagnuolo, I. Tedesco, S. Bilotto, and G.L. Russo. 2012. The flavonoid quercetin in disease prevention and therapy: Facts and fancies. Biochem. Pharmacol. 83:6-15.

Septiningsih, E.M., A.M. Pamplona, D.L. Sanchez, C.N. Neeraja, G.V. Vergara, S. Heuer, A.M. Ismail, and D.J. Mackill. 2009. Development of submergence-tolerant rice cultivars: The Sub1 locus and beyond. Ann. Bot. 103:151-160.

Shirley, B.W. 1996. Flavonoid biosynthesis: 'New' functions for an 'old' pathway. Trends Plant Sci. 1:377-382.

Slimestad, R., T. Fossen, and I.M. Vågen. 2007. Onions: A source of unique dietary flavonoids. J. Agric. Food Chem. 55:10067-10080.

Spelt, C., F. Quattrocchio, J.N. Mol, and R.E. Koes. 2000. Anthocyaninl of petunia encodes a basic helix-loop-helix protein that directly activates transcription of structural anthocyanin genes. Plant Cell 12:1619-1631.

Varshney, R.K., S.N. Nayak, G.D. May, and S.A. Jackson. 2009. Next-generation sequencing technologies and their implications for crop genetics and breeding. Trends Biotechnol. 27:522-530.

Veitch, N.C. and R.J. Grayer 2011. Flavonoids and their glycosides, including anthocyanins. Nat. Prod. Rep. 28:1626-1695.

Vogt, T. 2010. Phenylpropanoid biosynthesis. Mol. Plant 3:2-20. Yamazaki, M., Y. Makita, K. Springob, and K. Saito 2003. Regulatory mechanisms for anthocyanin biosynthesis in chemotypes of Perilla frutescens var. crispa. Biochem. Eng. J. 14:191-197. 